\title{
Depth-selection behavior and longevity in Daphnia: an evolutionary test for the predation-avoidance hypothesis
}

\author{
Piotr Dawidowicz • Piotr Prędki • \\ Barbara Pietrzak
}

Received: 30 March 2012/Accepted: 5 November 2012/Published online: 23 November 2012

(C) The Author(s) 2012. This article is published with open access at Springerlink.com

\begin{abstract}
The classic evolutionary theory of senescence predicts that long lifespan evolves under low risk of extrinsic mortality. As lakes present planktonic animals with vertical gradients of mortality risk associated with fish predation, we expected the individual lifespan of Daphnia of the "hypolymnetic" clones to be longer than that of "epilimnetic" Daphnia. In order to test this prediction, we performed a laboratory study on 14 clones from the $D$. longispina species complex, taken during the daytime either from epilimnion or hypolimnion of three mesotrophic lakes. "Epilimnetic" Daphnia started reproduction earlier, aged faster, and lived shorter than their "hypolimnetic" conspecifics. Our results indirectly support the predation-avoidance hypothesis as the ultimate explanation for depth-selection behavior in Daphnia.
\end{abstract}

Guest editors: Marina Manca \& Piet Spaak / Cladocera: Proceedings of the 9th International Symposium on Cladocera

P. Dawidowicz $(\bowtie) \cdot$ P. Prędki · B. Pietrzak

Department of Hydrobiology, Institute of Zoology,

Warsaw University, Banacha 2, 02-097 Warsaw, Poland

e-mail: p.dawidowicz@uw.edu.pl

Present Address:

B. Pietrzak

Max Planck Institute for Demographic Research,

Laboratory of Evolutionary Demography,

Konrad-Zuse-Straße 1, 18057 Rostock, Germany
Keywords Daphnia longispina complex · Evolution of senescence - Surface-avoidance behavior · Predation risk · Extrinsic mortality

\section{Introduction}

Depth-selection behavior in zooplankton, or the diel patterns thereof, known as diel vertical migrations (DVM), are considered a strategy to reduce lightdependent mortality imposed by fish (Zaret and Suffern, 1976; Gliwicz, 1986). Surface-avoiding animals make a trade off, whereby mortality due to predation is reduced at the expense of a decrease in metabolic rates and reproductive output brought about by the low temperature and scarcity of food in deepwater habitats (e.g., Swift, 1976; Dawidowicz and Loose, 1992).

According to the evolutionary theory of senescence (ETS), in populations exposed to high extrinsic mortality short lifespan and great investments in early-life reproduction should evolve, as the contribution of later ages to overall fitness in such populations is reduced. In contrast, low extrinsic mortality selects for longer lifespan and delayed reproduction (Medawar, 1952; Williams, 1957; Hamilton, 1966). Although these general patterns do not hold for all species (e.g., Reznick et al. 2004, and further discussion by Abrams, 2004), life-table studies performed on Daphnia by Dudycha \& Tessier (1999) and Dudycha (2001) support the predictions of classic ETS. They have analyzed senescence patterns in populations of Daphnia pulex-pulicaria 
inhabiting enivironments dubbed "risky" (i.e., temporary ponds) or "safe" (deep lakes). When compared with the clones from lakes, Daphnia isolated from ponds indeed invested more in early-life fitness at the cost of fast senescence and reduced lifespan.

However, deep-lake habitats cannot be treated as uniform where the distribution of the mortality risk is concerned. The well-lit surface waters offer a foraging site for planktivorous fish that search less and less efficiently for prey at ever greater depths with their attenuated illumination (e.g., Vinyard and O'Brien, 1975). Consequently, a given lake should be characterized by a gradient as regards the danger of predation-from habitats that are risky (near the surface) to those considered safe (in deep water). As the genetic component to depth-selection behavior in Daphnia is strong (e.g., Weider, 1984, Seda et al., 2007), this risk-gradient should translate into divergent longevity among Daphnia clones that differ in their daytime depth preferences.

In consequence, the main goal of this study has been to test the prediction that Daphnia from deepdwelling clones of the $D$. longispina species complex live longer and start reproduction later than individuals from the clones that prefer to stay in shallow waters during the day. Considering the ETS arguments, this would support the ultimate explanation of surface-avoidance behavior in zooplankton in terms of the avoidance of extrinsic mortality (predation).

\section{Materials and methods}

We used 14 clones from the Daphnia longispina species complex, from mesotrophic lakes Garbaś, Babięty, and Hańcza in north-eastern Poland. The lakes are relatively large (surface area 140, 264, and 311 ha, respectively) and deep (48, 65, and $108 \mathrm{~m}$, respectively) and isolated from each other (the minimum distance between the lakes is $18 \mathrm{~km}$ ). The lakes host rich planktivorous fish communities, with roach (Rutilus rutilus), bream (Abramis brama), perch (Perca fluviatilis), smelt (Osmerus eperlanus), and whitefish (Coregonus albula) being dominant species. Among invertebrate predators the cyclopoid copepods predominate, with cladoceran Leptodora kindtii and Chaoborus larvae being very scarce or absent (M. Ślusarczyk, personal communication). The Daphnia were collected at noon at the end of the summer stratification period (the thermocline between 8 and $18 \mathrm{~m})$, with a closing plankton net (150 $\mu \mathrm{m}$ mesh size) towed vertically through the hypolimnion (from 30 to $20 \mathrm{~m}$ depth) or epilimnion (from depth of $8 \mathrm{~m}$ to the surface). Only the mature (egg carrying) females longer than $1.5 \mathrm{~mm}$ were isolated from the samples to establish laboratory clones. Six "epilimnetic" clones and eight "hypolimnetic" clones were maintained in laboratory batch cultures at room temperature $\left(20-24^{\circ} \mathrm{C}\right)$ for 3 months prior to the study. The taxonomic position of each clone was diagnosed using electrophoretic analysis of AAT, AO, PGN, and GPI isoenzymes (Wolf and Mort, 1986; see Hebert and Beaton, 1989, for the electrophoresis protocol).

Water for the culturing of Daphnia originated from the small, eutrophic Lake Szczęśliwice in Warsaw. It was prefiltered through a $1-\mu \mathrm{m}$ sieve and stored in a large, aerated glass tank for 4 weeks before use. In order to obtain the culture medium, the water was filtered again through a $0.45 \mu \mathrm{m}$ membrane and enriched with laboratory-grown Scenedesmus obliquus to the desired concentration of $1 \mathrm{mg} \mathrm{C} \mathrm{L}^{-1}$.

Longevity was tested within cohorts of 30 Daphnia from each clone, with an initial age of $12 \pm 6 \mathrm{~h}$, these being individually located in $100 \mathrm{ml}$ glass beakers filled with the culture medium. The beakers were kept in a laboratory at constant temperature $\left(23 \pm 1^{\circ} \mathrm{C}\right)$ and summer photoperiod (16:8 L:D). The setup was inspected daily and live individuals were counted and transferred into new beakers with fresh medium. The offspring (if present) were removed from the culture. The experiment lasted 62 days. Median longevity and median age at the first reproduction (i.e., time of release of first neonates from the brood chamber) were determined. The number of offspring released within first 10 days of life of the Daphnia served as an arbitrary measure of their early reproductive effort. In order to determine patterns of age-specific fitness in each investigated clone, we calculated the so-called intrinsic value $i_{x}$, which describes a contribution of each age class $(x)$ to the total lifetime reproduction, according to Dudycha and Tessier (1999):

$i_{x}=\sum_{j=x}^{\infty} l_{j} m_{j} / \sum_{j=0}^{\infty} l_{j} m_{j}$,

where $l_{j}$ is cumulative survivorship and $m_{j}$ is per capita fecundity. The median age at the $50 \%$ value of agespecific fitness was determined. The significance of differences between the above parameters determined 
for "epilimnetic" and "hypolimnetic" clones was tested with the non-parametric Mann-Whitney statistics.

\section{Results}

The median lifespan of Daphnia longispina individuals of the "epilimnetic" clones was 54\% that of the "hypolimnetic" ones (Fig. 1; Table 1). Age-specific fitness, as expressed by the intrinsic value $\left(i_{x}\right)$, decreased significantly faster in Daphnia collected at the surface, than in clones isolated from the hypolimnion (Fig. 2; Table 1). These patterns were accompanied by earlier maturation and larger number of offspring released within the first 10 days of their life by "epilimnetic" clones than by "hypolimnetic" ones (Table 1).

\section{Discussion}

Despite the considerable phenotypic plasticity of habitat choice patterns in Daphnia (Pijanowska, 1993), genetic polymorphism in depth preferences is tangible, producing interclonal differences in vertical distribution and migration patterns (Weider, 1984; De Meester et al., 1994). We found that clones (genotypes) of Daphnia that stayed deep in the water column started reproduction later, aged relatively slowly, and lived longer than Daphnia of surfacedwelling clones. The Daphnia were collected at the end of summer stratification period, in October, i.e., at least 6 months after hatching of the overwintering ephippia. Thus, even if the studied populations were panmictic (during sexual reproduction period a year earlier) there was long time for clonal selection to eliminate the genotypes mismatching behavioral (habitat choice) and life-history (size at first reproduction, longevity) traits. Although co-adaptation between depth-selection behavior and certain lifehistory traits, such as size at birth or size/age at the first reproduction, has been reported (Tessier and Leibold, 1997; Sakwińska and Dawidowicz, 2005), the association between surface avoidance and longevity in Daphnia has not been demonstrated before.

It seems unlikely that the positive association between the residence depth and lifespan of particular clones could be driven by a genotype-habitat interaction. The temperature conditions of the survival test $\left(23^{\circ} \mathrm{C}\right)$ were closer to those of the near-surface habitats, yet individuals of the "hypolimnetic" clones, most likely adapted to deep and cold waters, lived longer than those of the "epilimnetic" clones. All the clones in this study were tested for longevity at the same, fixed temperature. Thus the observed variation in lifespan among clones was not an effect of different ambient temperatures at the depths they would prefer and, consequently, different metabolic rates of the animals.

The evolution of links between the depth preferences of planktonic animals and traits that affect their vulnerability to predation (such as size at maturation) was likely compelled by vertical gradients in predation risk. The existence of such genetic correlations
Fig. 1 Survival curves for the "epilimnetic" (thin line, open symbols) and "hypolimnetic" (thick line, filled symbols) Daphnia longispina clones

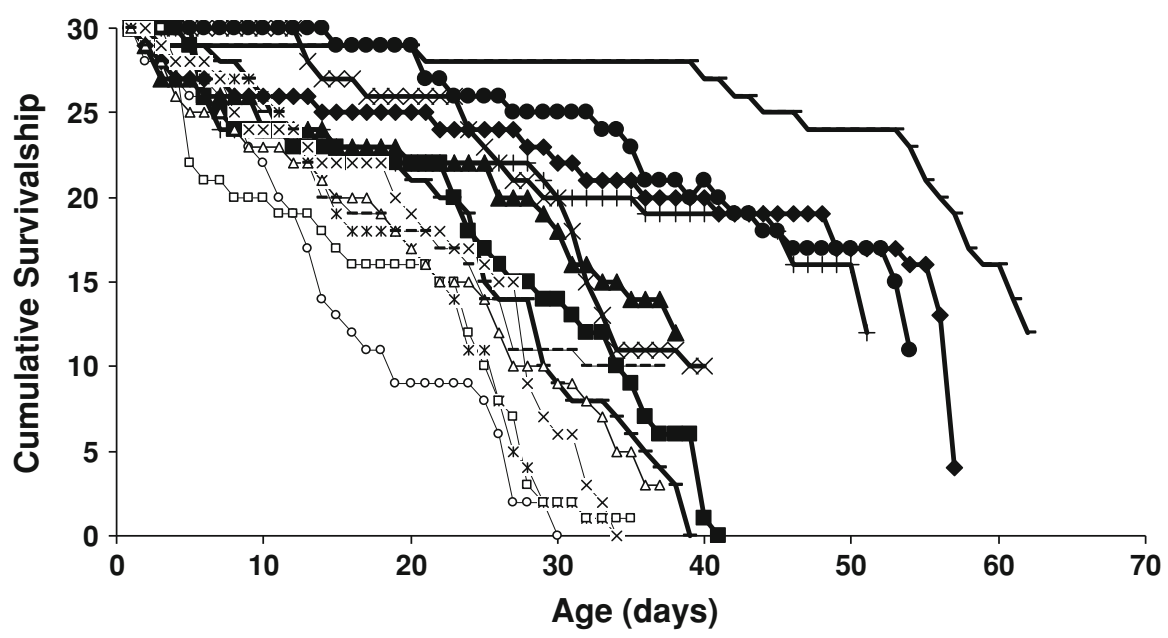


Table 1 Life-history traits of $D$. longispina of "hypolimnetic" and "epilimnetic" clones

\begin{tabular}{llll}
\hline & $\begin{array}{l}\text { Epilimnetic } \\
\text { clones }(n=6)\end{array}$ & $\begin{array}{l}\text { Hypolimnetic clones } \\
(n=8)\end{array}$ & $P$ (Mann-Whitney) \\
\hline Median longevity (days) & 23.0 & 42.5 & 0.001 \\
Median age of 50\% age-specific fitness (days) & 15.0 & 23.5 & 0.001 \\
Median age at first reproduction (days) & 7.0 & 9.0 & 0.021 \\
Mean no. of offspring in first 10 days ( $\pm 1 \mathrm{SD})$ & $3.07 \pm 1.12$ & $1.46 \pm 1.47$ & 0.029 \\
\hline
\end{tabular}

Fig. 2 Age-specific fitness (as expressed by intrinsic value $i_{x}$ ) curves for the "epilimnetic" (thin line, open symbols) and "hypolimnetic" (thick line, filled symbols) Daphnia longispina clones. The same symbols on Fig. 1 refer to the same clones

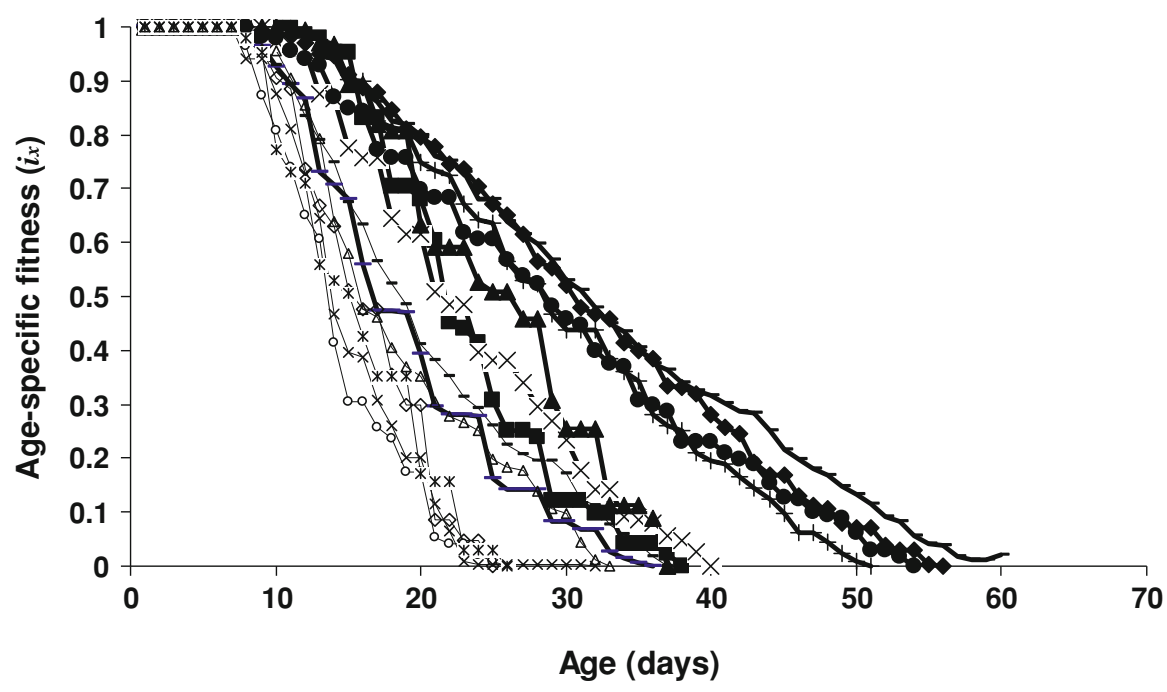

provides a commonly invoked argument in favor of predator avoidance as the ultimate explanation of DVM (Lampert, 1989). The positive association between the "physiological" lifespan in our Daphnia clones and their daytime residence depth is in accordance with the predator-avoidance hypothesis as well as with the classic evolutionary theory of senescence. Fish predation is the major source of mortality in large-bodied cladoceran zooplankton in lakes, prevailing over starvation, parasitism, or aging (Gliwicz and Pijanowska, 1989). Consequently, successful avoidance of fish favors low adult mortality, something that should in turn select for slow senescence (Williams, 1957; Hamilton, 1966). Deep-dwelling Daphnia clones are often exposed to predation by invertebrates, which themselves are forced to migrate extensively in the presence of fish (Ohman, 1990). These gape-limited predators prey upon small-sized zooplankton, including juvenile Daphnia (Zaret, 1980). Poor juvenile survival leads to a relative increase in the contribution of late age classes to lifetime fitness and, consequently, should further enhance selection for slower aging (Abrams, 1993).

A diversity of depth-selection behaviors could be the underlying cause of the polymorphism in body size in cladoceran populations. The Daphnia that reside in deep water attain large size, not only because the current selection by planktivorous fish is weak in their habitats, but also because they are physiologically capable of living longer and growing larger.

Acknowledgments We thank Tomasz Brzeziński for his help in the analysis of izoenzymes and Joanna Pijanowska and Maciej Gliwicz for comments on the previous version of the manuscript. This study was supported by grant IP 2010031670 from Polish Ministry of Science and higher Education to Barbara Pietrzak.

Open Access This article is distributed under the terms of the Creative Commons Attribution License which permits any use, distribution, and reproduction in any medium, provided the original author(s) and the source are credited. 


\section{References}

Abrams, P. A., 1993. Does increased mortality favor the evolution of more rapid senescence? Evolution 49: 10551066.

Abrams, P. A., 2004. Mortality and lifespan. Nature 431: 1048-1049.

Dawidowicz, P. \& C. J. Loose, 1992. Metabolic costs during diel vertical migration in Daphnia. Limnology and Oceanography 37: 665-669.

De Meester, L., J. Vandenberghe, K. Desender \& H. Dumont, 1994. Genotype-dependent daytime vertical-distribution of daphnia-magna in a shallow pond. Belgian Journal of Zoology 124: 3-9.

Dudycha, J. L., 2001. The senescence of Daphnia from risky and safe habitats. Ecology Letters 4: 102-105.

Dudycha, J. L. \& A. J. Tessier, 1999. Natural genetic variation of life span, reproduction, and juvenile growth in Daphnia. Evolution 53: 1744-1756.

Gliwicz, Z. M., 1986. Predation and the evolution of vertical migration in zooplankton. Nature 343: 638-640.

Gliwicz, Z. M. \& J. Pijanowska, 1989. The role of predation in zooplankton succession. In U. Sommer, (ed.), Plankton Ecology. Succession in Plankton Communities. Springer, Berlin: 253-296.

Hamilton, W. D., 1966. The molding of senescence by natural selection. Journal of Theoretical Biology 12: 12-45.

Hebert, P. D. N. \& M. J. Beaton, 1989. Methodologies for allozyme analysis using cellulose acetate electrophoresis. Helena Laboratories, Beaumont, TX: 1-31.

Lampert, W., 1989. The adaptive significance of diel vertical migration of zooplankton. Functional Ecology 3: 21-27.

Medawar, P. B., 1952. An unsolved problem in biology. Lewis, London.

Ohman, M. D., 1990. The demographic benefits of diel vertical migration by zooplankton. Ecological Monographs 60: 257-281.
Pijanowska, J., 1993. Diel vertical migration in zooplankton: fixed or inducible behaviour? Archive für Hydrobiologie, Beiheft Ergebnisse der Limnologie 39: 89-97.

Reznick, D. N., M. J. Bryant, D. Roff, C. K. Ghalambor \& D. E. Ghalambor, 2004. Effect of extrinsic mortality on the evolution of senescence in guppies. Nature 431: 10951099.

Sakwińska, O. \& P. Dawidowicz, 2005. Life history strategy and depth selection behavior as alternative antipredator defenses among natural Daphnia hyalina populations. Limnology and Oceanography 50: 1284-1289.

Seda, J., K. Kolarova, A. Petrusek \& J. Machacek, 2007. Daphnia galeata in the deep hypolimnion: spatial differentiation of a "typical epilimnetic" species. Hydrobiologia 594: 47-57.

Swift, M. C., 1976. Energetics of diel vertical migration in Chaoborus trivitatus larvae. Ecology 57: 900-914.

Tessier, A. J. \& M. A. Leibold, 1997. Habitat use and ecological specialization within lake Daphnia populations. Oecologia 109: 561-570.

Vinyard, G. L. \& W. J. O’Brien, 1975. Effect of light and turbidity on the reactive distance of bluegill sunfish (Lepomis macrochirus). Journal of the Fisheries Research Board of Canada 32: 1860-1863.

Weider, L. J., 1984. Spatial heterogeneity of Daphnia genotypes: vertical migration and habitat partitioning. Limnology and Oceanography 29: 225-235.

Williams, G. C., 1957. Pleiotropy, natural selection and the evolution of senescence. Evolution 11: 398-411.

Wolf, H. G. \& M. A. Mort, 1986. Inter-specific hybridization underlies phenotypic variability in Daphnia populations. Oecologia 68: 507-511.

Zaret, T. M., 1980. Predation and Freshwater Communities. Yale Univ Press, New Haven.

Zaret, T. M. \& J. S. Suffern, 1976. Vertical migration in zooplankton as a predator avoidance mechanism. Limnology and Oceanography 21: 804-813. 